\title{
Common Nasal Anomalies and Its Implications on Intubation in Head and Neck Surgeries
}

\author{
Ganesh Elumalai $^{1,}$,, Sushma Chodisetty ${ }^{2}$, Sanjoy Sanyal $^{3}$ \\ ${ }^{1}$ Department of Anatomy \& Neuroscience, College of Medicine, Texila American University, Georgetown, South America \\ ${ }^{2}$ Department of Casualty, National Medical College and Teaching Hospital, Tribhuvan University, Birgunj, Nepal \\ ${ }^{3}$ Department of Neuroscience, College of Medicine, Texila American University, Georgetown, South America
}

Email address:

ganesh.e@tau.edu.gy (E. Ganesh)

${ }^{*}$ Corresponding author

\section{To cite this article:}

Ganesh Elumalai, Sushma Chodisetty, Sanjoy Sanyal. Common Nasal Anomalies and Its Implications on Intubation in Head and Neck Surgeries. Journal of Surgery. Vol. 4, No. 4, 2016, pp. 81-84. doi: 10.11648/j.js.20160404.11

Received: June 16, 2016; Accepted: June 24, 2016; Published: July 18, 2016

\begin{abstract}
Nasotracheal intubation used to be the preferred route for prolonged intubation in critical care units. Nasotracheal intubation may sometimes cause nasal trauma. The study included one hundred and nine (109) adult patients, were scheduled for elective head and neck surgeries with general anaesthesia, requiring nasotracheal intubation. All the patients compiled the criteria of American Society of Anesthesiologists (ASA) physical status I and II were included in this study. The incidence of complicated laryngoscopy was assumed as $8 \%$, confidence levels at $99 \%$ and an error of $3 \%$, the total sample size were One hundred and three (103) patients. Observation, recorded bleeding 63.11\% (65-patients) of the time with the literature stating epistaxis rates from $17-77 \%$. The study found there was a significant relationship of soft tissue profile and number of intubation attempts. In this study a concave profile was more likely to have multiple attempts, there was a significant relationship between moderate and severe bleeding and number of intubation attempts and in $04.85 \%$ (05-patients) of those patients with severe bleeding there were multiple intubation attempts. Thyromental distance and Mallampati score did not seem to have a significant relationship with either the number of intubation attempts or severity of bleeding. This may demonstrate that multiple attempts led to an increase in bleeding due to increased trauma or that bleeding from the nose into the oropharnyx and hypo-pharynx contributed to a difficult view of the larynx for passing the tube between the cords. The clinical relevance from this study to create an algorithm or define a set of factors to alert anesthetists to aware of knowledge about the common nasal anomalies for the difficult nasotracheal (NT) intubation.
\end{abstract}

Keywords: Nasotracheal Intubation, Dental Surgeries, Oral Surgeries, Nasal Anomalies

\section{Introduction}

Nasotracheal intubation (NTI) is one of the most common methods used to incite anaesthesia for emergency surgeries in the head and neck region, when orotracheal intubation is not feasible. Nasotracheal intubation used to be the preferred route for prolonged intubation in critical care units. Nasotracheal intubation may sometimes cause nasal trauma [1]. The most frequent complication is epistaxis, which may be occasionally troublesome, even with vasoconstrictors, with a smaller, softened and well-lubricated tube with careful manipulations. More seriously, there have been reports of partial and even total avulsions of middle and inferior turbinate's [2, 3, 4, 5]. More rarely, an amputated turbinate may obstruct the nasotracheal tube, further endangered the patient safety [6].

In nasotracheal intubation, there are two basic anatomical pathways in the nostril, through which the endotracheal tube might pass [7]. The upper pathway lies between the inferior turbinate and middle turbinate. Lower pathway lies along the floor of the nose and is more secure. The lower pathway is a preferred pathway. Intranasal abnormalities are seen in twothird of oral surgery patients [8]. Anatomical variations like nasal-concha, bulla-ethmoidalis, nasal-septal deviations, nasal-spur and nasal-polyps, are common unilateral obstructions may affects the intubation [9]. The knowledge 
about the common anatomical nasal anomalies is more helpful to understand the nasal variations. The preanaesthetic diagnostic evaluations are more helpful to prevent the complications.

\section{Materials and Methods}

One hundred and nine (109) adult patients were scheduled for elective head and neck surgeries with general anaesthesia, requiring nasotracheal intubation. All the patients compiled the criteria of American Society of Anesthesiologists (ASA) physical status I and II were included in this study. The study considered all the aspects of human care compiled with the ethical guidelines and technical requirements were approved by the Institutional Human Ethics Committee (IHEC) and Institutional Review Board (IRB), National Medical College and Teaching Hospital. The incidence of complicated laryngoscopy was assumed as $8 \%,[10]$ confidence levels at $99 \%$ and an error of $3 \%$, the total sample size were One hundred and three (103) patients (Table-1).

Table 1. Pre-Anesthetic Classification of patients under Modified Mallampati class (MMC) [11].

\begin{tabular}{llll}
\hline MODIFIED MALLAMPATI CLASSIFICATION & & \\
\hline CLASS & DESCRIPTION & NUMBER OF SUBJECTS (n=103) & PERCENTAGE OF SUBJECTS (\%) \\
\hline 0 & Ability to see any part of the epiglottis upon & 02 & 01.94 \\
I & mouth opening and tongue protrusion & & 56.31 \\
II & Soft palate, fauces, uvula, pillars visible & 58 & 21.35 \\
III & Soft palate, fauces, uvula visible & 22 & 12.62 \\
IV & Soft palate, base of uvula visible & 13 & 07.76 \\
\hline
\end{tabular}

In the operating room, the patient was placed in the supine position with standard monitors applied (electrocardiogram, noninvasive blood pressure, pulse oximetry, capnography and temperature). General anesthesia was induced with proper ventilatory support. An appropriate sized endotracheal tube (ET) was selected for the patient, using the formula, ((Age of patient in years $/ 4)+4)=$ diameter of endotracheal tube in millimeters". The chosen endotracheal tube was lubricated and inserted into the right naris at room temperature. If the endotracheal tube met resistance a second tube, one half sizes smaller, was taken and introduced. This procedure was repeated as required until a tube could be gently passed into the nasopharynx. During this procedure, the laryngeal inlet was visualized through direct laryngoscopy (DL) and the endotracheal tube was placed in the appropriate position using the forceps. The ET tube was secured in a standard manner. The study ended after successful intubation of endotracheal tube, it was confirmed by the assessment of chest movement, auscultation and capnography.

To achieve the successful intubation, the posterior oropharnyx was visualized with DL, before the chosen endotracheal tube (ET) tube was passed through the vocal cords. The epistaxis was rated as no, mild, moderate and severe. "No epistaxis" indicated with no bleeding, "mild epistaxis" represented as evidence of blood in the endotracheal tube (ET) tube, "moderate epistaxis" indicated the endotracheal tube (ET) tube is splashed obviously with the blood, and "severe epistaxis" represented with a active bleeding or accumulation of blood in the posterior oropharnyx, was sufficient to impede the intubation.

The number of intubation attempts, amount of epistaxis and number of times a new tube was chosen were recorded. Student's t-test, used to analyze the significant differences between easy and difficult laryngoscopy and the number of intubation attempts in relations to the Soft tissue, Cricoid pressure, Epistaxis and preferential Naris.

\section{Observations}

The significant airway differences between single attempt and multiple attempts laryngoscopy were assessed by: (1) Modified Mallampati class (MMC) [11]; of oropharyngeal view (Table-2); (2) Inter-incisor distance (IID), with the mouth fully opened; (3) Thyromental distance (TMD); (4) Sternomental distance (SMD) obtained by measuring the straight distance from thyroid notch and sternal notch, with the head in extension; (5) Range of head and neck movements described by Wilson et al [12]; (6) Mandibular protrusion limitation; (a). the lower incisors can be brought in front of the upper incisors or (b). the lower incisors can be advanced only to the level of upper incisors or (c). cannot reach the level of the upper incisors; (7) Ratio of height (cms) and Thyromental distance (TMD) (cms) [RHTMD ratio]; (8) Dentition: Loose, missing, protruding teeth or edentulous were noted.

Table 2. Modified Mallampati classification differences between Easy and Difficult Laryngoscopy.

\begin{tabular}{|c|c|c|c|}
\hline \multicolumn{4}{|c|}{ MODIFIED MALLAMPATI CLASSIFICATION DIFFERENCES BETWEEN EASY AND DIFFICULT LARYNGOSCOPY } \\
\hline \multirow{2}{*}{ CLASS } & \multirow{2}{*}{ DESCRIPTIONS } & \multicolumn{2}{|c|}{ LARYNGOSCOPY $(n=103)$} \\
\hline & & Single Intubation $(\mathrm{n}=\mathbf{8 2})$ & Multiple Intubation $(n=21)$ \\
\hline 0 & $\begin{array}{l}\text { Ability to see any part of the epiglottis upon mouth opening } \\
\text { and tongue protrusion }\end{array}$ & $02(01.94 \%)$ & $0(0 \%)$ \\
\hline I & Soft palate, fauces, uvula, pillars visible & $52(50.48 \%)$ & $06(05.82 \%)$ \\
\hline II & Soft palate, fauces, uvula visible & $15(14.56 \%)$ & $07(06.79 \%)$ \\
\hline III & Soft palate, base of uvula visible & $08(07.76 \%)$ & $05(04.85 \%)$ \\
\hline IV & Soft palate not visible at all & $05(04.85 \%)$ & $03(02.91 \%)$ \\
\hline
\end{tabular}


The present study demonstrates that, TMD in the multiple attempts laryngoscopy group was significantly shorter $(5.8+1.0)$ to compare with the single attempt $(6.3+0.9)$ laryngoscopy group. In this study the RH-Ratio of height (cm) and TMD- Thyromental distance (cm) - (RHTMD), was significantly higher in patients in the multiple attempts laryngoscopy group $(28.5+5.3)$ compared with that in the single attempt laryngoscopy group $(22.6+3.2)$. Sternomental distance (SMD) is an indicator of head and neck mobility [13]. There was a statistically significant difference between SMD in the single attempt and multiple attempts laryngoscopy groups $(14.3+1.2 \mathrm{~cm}$ and $13.5+2.3 \mathrm{~cm}$, respectively). The other parameters like Inter-incisor distance (IID) $<3.5 \mathrm{cms}$, Range of head and neck movement $<80^{\circ}$ and Dentition were noticed in negligible, but, in statistical significant ratio (Table-3).

Table 3. Significant Airway differences between Easy and Difficult Laryngoscopy.

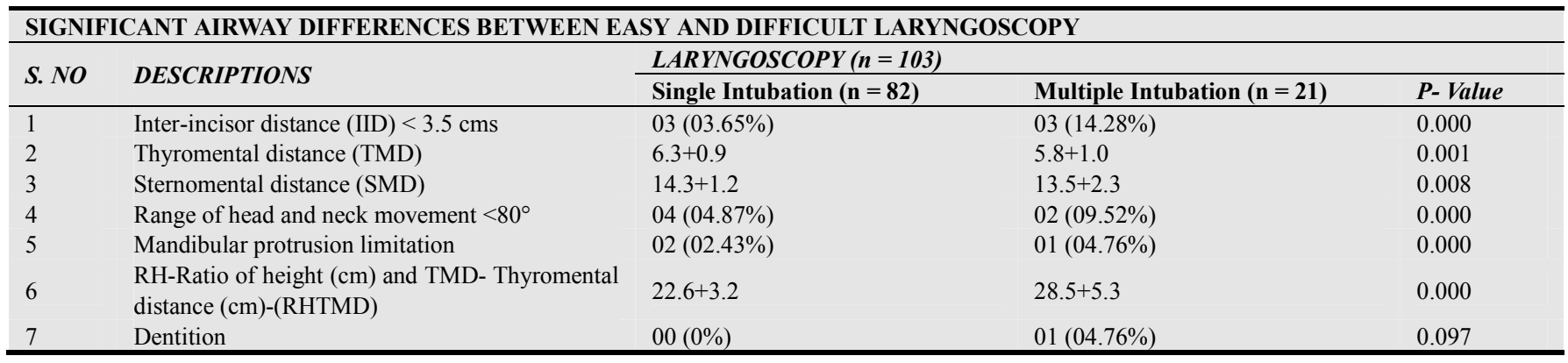

Values are mean $+\mathrm{SD}$ and numbers (percent)

Current study observed that there was a significant association between the soft tissue profile and the number of intubation attempts made for the secure airway. Patients required single intubation attempt had the highest convex soft tissue profile as $95.18 \%$ and a concave soft tissue profile were recorded as lowest ratio like $21.42 \%$ in the presented cases. However, for the patients required multiple intubation attempts, the highest ratio of $78.57 \%$ had the convex soft tissue profile and the lowest ratio like $04.81 \%$ were recorded with concave soft tissue profile $(p=0.0284)$, (Table-4). The study recorded that, for the patients who were intubated on the first attempt $96 \%$ received no Cricoid pressure. However for those who required multiple intubation attempts, $21.42 \%$ of patients received the application of Cricoid pressure $(p=0.0038)$, (Table-4). On epistaxis, the patients intubated successfully on the first attempt like $97.36 \%$ had no bleeding and $96 \%$ had mild bleeding. However, the patients needed multiple intubation attempts $29.41 \%$ demonstrated severe bleeding $(p=0.0022)$. Further analysis revealed that the number of intubation attempts held no statistically significant relationships to any other categorical variables tested. In conclusion, $08.69 \%$ of those patients had moderate epistaxis on multiple intubation attempts, while only $02.63 \%$ of patients presents with the absence of epistaxis in multiple attempts. ( $\mathrm{P}=0.0048)$, (Table-4). Where single intubation attempt was made the right naris was used $89.41 \%$ of the time, however for those requiring multiple intubation attempts $77.77 \%$ of the successful intubations took place via the left naris $(p=0.0002)$. As a result patients for whom the left naris was used for intubation had higher odds of having multiple intubation attempts compared to patients who were intubated through the right naris, (Table-4).

Table 4. Anatomical Variations induce the number of Intubation attempts.

\begin{tabular}{|c|c|c|c|c|c|c|}
\hline \multicolumn{7}{|c|}{ ANATOMICAL VARIATIONS INDUCE THE NUMBER OF INTUBATION ATTEMPTS } \\
\hline S. NO & Major Variable factors & Sub factors & Frequency & Single Intubation & Multiple Intubation & P-Value \\
\hline \multirow{3}{*}{1} & \multirow{3}{*}{ Soft tissue } & Concave & $14(13.59 \%)$ & $03(21.42 \%)$ & $11(78.57 \%)$ & \\
\hline & & Convex & $83(80.58 \%)$ & $79(95.18 \%)$ & $04(04.81 \%)$ & 0.0284 \\
\hline & & Straight & $05(04.85 \%)$ & $04(80.00 \%)$ & $01(20.00 \%)$ & \\
\hline \multirow{2}{*}{2} & \multirow{2}{*}{ Cricoid pressure } & Used & $28(27.18 \%)$ & $22(78.57 \%)$ & $06(21.42 \%)$ & \\
\hline & & Not used & $75(72.81 \%)$ & $72(96.00 \%)$ & $03(04.00 \%)$ & 0.0038 \\
\hline \multirow{3}{*}{3} & \multirow{3}{*}{ Epistaxis } & Mild Bleeding & $25(24.27 \%)$ & $24(96.00 \%)$ & $01(04.00 \%)$ & \\
\hline & & Moderate Bleeding & $23(22.33 \%)$ & $21(91.30 \%)$ & $02(08.69 \%)$ & \\
\hline & & Severe Bleeding & $17(16.50 \%)$ & $12(70.58 \%)$ & $05(29.41 \%)$ & \\
\hline \multirow{2}{*}{4} & \multirow{2}{*}{ Preferential Naris } & Right & $85(82.52 \%)$ & $76(89.41 \%)$ & $09(10.58 \%)$ & 0.0002 \\
\hline & & Left & $18(17.47 \%)$ & $04(22.22 \%)$ & $14(77.77 \%)$ & \\
\hline
\end{tabular}

\section{Discussion}

The current bleeding rates were consistent with the current literature findings for nasotracheal (NT) intubation and epistaxis, recorded bleeding $63.11 \%$ (65-patients) of the time with the literature stating epistaxis rates from $17-77 \%[14$, 15]. The study found that, there was a significant relationship 
of soft tissue profile and number of intubation attempts. In this study, a concave profile was more likely to have multiple attempts, there was a significant relationship between moderate and severe bleeding and number of intubation attempts and in $04.85 \%$ (05-patients) of those patients with severe bleeding there were multiple intubation attempts. The fact that a concave profile had a significant relationship with the number of intubation attempts differs from most studies concerning oral intubations. Thyromental distance and Mallampati score did not seem to have a significant relationship with either the number of intubation attempts or severity of bleeding. Interestingly these two assessments, along with soft tissue profile and maximum opening continue as the gold standards for assessing airways. This may demonstrate that multiple attempts led to an increase in bleeding due to increased trauma or that bleeding from the nose into the oropharnyx and hypo-pharynx contributed to a difficult view of the larynx for passing the tube between the cords. The present study encountered multiple intubation attempts. As mentioned before, multiple intubations attempts are already a known issue for causing epistaxis. Future studies should further understand if the multiple intubation attempts were with the same tube. Also there was a significant relationship of which naris was used for intubation. Using the left naris for intubation was statistically significant possibly due to the type of nasotracheal (NT) tube used. The tubes used were Mallinckrodt and designed to be introduced into the right side. It was controlled by using Parker tubes right versus left would not have been a factor [16]. This was the first study of its nature in terms of looking at nasotracheal intubation. Compared to other studies, it had an appropriate sample size but did not have inter/intra rater reliability that the other studies had. The unique to this study was successful in finding the parameters, which appeared to be correlated with multiple attempts.

\section{Conclusion}

The clinical relevance from this study to create an algorithm or define a set of factors to alert anesthetists to aware of knowledge about the common nasal anomalies for the difficult nasotracheal (NT) intubation. Ultimately it would allow an anesthesiologist to decide what is best for the patient and decide against using a nasotracheal (NT) tube. Future considerations for this study would include increasing the sample size to strengthen the conclusion of this study. Further studies might better understand the relationship of cricoid pressure and if it contributed to multiple intubation attempts or if the act of providing cricoid pressure caused multiple attempts. Additionally, using radiographic imaging such as cephelometric radiograph or orthopantogram may better allow us to accurately understand the skeletal relationships of patients versus a subjective assessment of a patient's soft tissue profile. It would also be helpful to understand how bleeding in the posterior oropharnyx impeded the view of the vocal cords and contributed to a difficult intubation.

\section{References}

[1] Bainton CR. Complications of managing the airway. In: Benumof JL, ed. Airway Management: Principles and Practice. St Louis: Mosby, 1995; 888.

[2] Scamman FL, Babin R. W. An unusual complication of nasotracheal intubation. Anesthesiology 1983; 59: 352-3.

[3] Wilkinson JA, Mathis RD, Dire DJ. Turbinate destruction: a rare complication of nasotracheal intubation. J Emerg Med 1986; 4: 209.

[4] Cooper R. Bloodless turbinectomy following blind nasal intubation. Anesthesiology 1989; 71: 469.

[5] Kuo MJ, Reid AP, Smith JE. Unilateral nasal obstruction: an unusual presentation of a complication of nasotracheal intubation. J Laryngol Otol 1994; 108: 991-2.

[6] Bandy DP, Theberge DM, Richardson DD. Obstruction of nasotracheal tube by inferior turbinate. Anesth Prog 1991; 38: $27-8$.

[7] Smith JE, Reid AP. Asymptomatic intranasal abnormalities influencing the choice of nostril for nasotracheal intubation. Br J Anaesth 1999; 83: 882-6.

[8] Smith JE, Reid AP. Asymptomatic intranasal abnormalities influencing the choice of nostril for nasotracheal intubation. B J Anaesth. 1999; 83: 882-886.

[9] Ahmed Nusrath A, Tong JL, Smith JE. Pathways through the nose for nasal intubation: a comparison of three endotracheal tubes. B J Anaesth. 2007; 100: 269-274.

[10] Prakash S, Rapsang AG, Mahajan S, Bhattacharjee S, Singh R, Gogia AR. Comparative Evaluation of the Sniffing Position with Simple Head Extension for Laryngoscopic View and Intubation Difficulty in Adults Undergoing Elective Surgery. Anesthesiol Res Pract. 2011. 2011297913.

[11] Samsoon GL, Young JR. Difficult tracheal intubation: A retrospective study. Anaesthesia. 1987;42: 487-90.

[12] Wilson ME, Spiegelhalter D, Robertson JA, Lesser P. Predicting difficult intubation. Br J Anaesth. 1988;61: 211-6.

[13] Ramadhani SA, Mohamed LA, Rocke DA, Gouws E. Sternomental distance as the sole predictor of difficult laryngoscopy in obstetric anaesthesia. Br J Anaesth. 1996;77: $312-6$.

[14] Tintinalli, Judith E., Claffey, James. Complications of nasotracheal intubation. Annals of Emergency Medicine Annals of Emergency Medicine, 1981; 10 (3): 142-144.

[15] Watt S, Pickhardt D, Lerman J, Armstrong J, Creighton PR, Feldman L. Telescoping tracheal tubes into catheters minimizes epistaxis during nasotracheal intubation in children. Anesthesiology. 2007; 106 (2): 238-42.

[16] Prior S, Heaton J, Jatana KR, Rashid RG. Parker flex-tip and standard-tip endotracheal tubes: A comparison during nasotracheal intubation. Anesthesia Progress. 2010; 57 (1): 18-24. 